\title{
The Black Hole Mass Distribution in the Galaxy
}

\section{Citation}

Özel, Feryal, Dimitrios Psaltis, Ramesh Narayan, and Jeffrey E. McClintock. 2010. "The Black Hole Mass Distribution in the Galaxy" The Astrophysical Journal 725 (2) (December 2): 19181927. doi:10.1088/0004-637x/725/2/1918.

\section{Published Version}

doi:10.1088/0004-637X/725/2/1918

\section{Permanent link}

http://nrs.harvard.edu/urn-3:HUL.InstRepos:25713853

\section{Terms of Use}

This article was downloaded from Harvard University's DASH repository, and is made available under the terms and conditions applicable to Other Posted Material, as set forth at http:// nrs.harvard.edu/urn-3:HUL.InstRepos:dash.current.terms-of-use\#LAA

\section{Share Your Story}

The Harvard community has made this article openly available.

Please share how this access benefits you. Submit a story.

Accessibility 


\title{
THE BLACK HOLE MASS DISTRIBUTION IN THE GALAXY
}

\author{
Feryal Özel ${ }^{1}$, Dimitrios Psaltis ${ }^{1}$, Ramesh Narayan ${ }^{2}$, and Jeffrey E. McClintock Mér $^{3}$ \\ ${ }^{1}$ Department of Astronomy, University of Arizona, 933 North Cherry Avenue, Tucson, AZ 85721, USA \\ ${ }^{2}$ Institute for Theory and Computation, Harvard University, 60 Garden Street, Cambridge, MA 02138, USA \\ ${ }^{3}$ Harvard-Smithsonian Center for Astrophysics, 60 Garden Street, Cambridge, MA 02138, USA \\ Received 2010 June 7; accepted 2010 October 25; published 2010 December 2
}

\begin{abstract}
We use dynamical mass measurements of 16 black holes in transient low-mass X-ray binaries to infer the stellar black hole mass distribution in the parent population. We find that the observations are best described by a narrow mass distribution at $7.8 \pm 1.2 M_{\odot}$. We identify a selection effect related to the choice of targets for optical follow-ups that results in a flux-limited sample. We demonstrate, however, that this selection effect does not introduce a bias in the observed distribution and cannot explain the absence of black holes in the $2-5 M_{\odot}$ mass range. On the high-mass end, we argue that the rapid decline in the inferred distribution may be the result of the particular evolutionary channel followed by low-mass X-ray binaries. This is consistent with the presence of high-mass black holes in the persistent, high-mass X-ray binary sources. If the paucity of low-mass black holes is caused by a sudden decrease of the supernova explosion energy with increasing progenitor mass, this would have observable implications for ongoing transient surveys that target core-collapse supernovae. Our results also have significant implications for the calculation of event rates from the coalescence of black hole binaries for gravitational wave detectors.
\end{abstract}

Key words: black hole physics - X-rays: binaries

Online-only material: color figures

\section{INTRODUCTION}

The distribution of stellar black hole masses in the Galaxy is intricately related to the population and evolution of massive stars, the energetics and dynamics of supernova explosions, and the dividing line between neutron stars and black holes. Inferring this distribution from observations helps address a number of outstanding questions in these areas.

Observations of mass losing evolved stars are one of the direct ways of studying the end stages of stellar evolution (Smartt 2009). Similarly, supernova and transient surveys (e.g., Rau et al. 2009, and references therein) provide increasingly larger data samples that have revealed a range of supernova properties and energetics that is wider than previously anticipated. Studies of the end products of supernova explosions, namely of the black hole and neutron-star mass distributions, provide a different and complementary approach to constraining models of massive stellar populations, evolution, and explosions (Timmes et al. 1996; Fryer 1999; Fryer et al. 2002; Woosley et al. 2002; Zhang et al. 2008).

The mass distribution of stellar black holes is a key ingredient in the calculation of the event rates for gravitational wave observatories such as LIGO, GEO500, and Virgo (Abadie et al. 2010). In addition, inferring this distribution may shed light on the origins and properties of pulsar-black hole binaries (Narayan et al. 1991), which are believed to be some of the best laboratories for strong-field gravity tests (Wex \& Kopeikin 1999).

During the last two decades, the masses of a large number of black holes in X-ray binaries have been measured (Remillard \& McClintock 2006; McClintock \& Remillard 2006), providing a sample suitable for the statistical inference of their parent mass distribution. Using this sample, we find in this article strong evidence for a very narrow distribution of masses for black holes in transient low-mass X-ray binaries. In particular, we show that there is a paucity of black holes with masses $\simeq 2-5 M_{\odot}$. Our results confirm and strengthen an earlier finding of Bailyn et al. (1998), who argued for a low-mass gap based on a limited sample of black holes. We explore in detail below known selection biases and demonstrate that the low-mass gap cannot be attributed to the observational selection of targets. We further argue that the lack of high-mass black holes in low-mass $\mathrm{X}$-ray binaries may result from a particular evolutionary path that leads to their formation.

\section{ACCRETING STELLAR-MASS BLACK HOLES AND BLACK HOLE CANDIDATES}

In Tables 1 and 2, we present basic data for all 23 confirmed black hole X-ray binaries (Remillard \& McClintock 2006). For the purpose at hand, we divide these systems into three groups: eight transient systems with orbital periods exceeding 1 day, nine shorter-period transient systems for which the measurement of black hole mass is problematic, and six systems with persistent $\mathrm{X}$-ray sources and massive O/B-type secondaries. In these tables, we provide the following data for most of the systems: Galactic coordinates, maximum X-ray intensity, orbital period, a distance estimate, mass function, mass ratio, inclination angle, and black hole mass. Our primary focus is on the 17 transient systems listed in Tables 1 and 2.

We summarize in Table 3 data for 32 additional transient systems that are believed to contain black hole primaries based on the spectral and timing properties of their X-ray sources. For each of these systems, we give both celestial and Galactic coordinates and the maximum X-ray intensity that has been reported.

\subsection{The 23 Black Hole Binaries}

We now survey the constraints that have been placed on the masses of the 23 confirmed stellar black holes via dynamical measurements. In determining the masses of black holes in 
Table 1

Properties of 23 Black Hole Binaries

\begin{tabular}{|c|c|c|c|c|c|c|c|c|c|}
\hline $\begin{array}{l}\text { Source } \\
\text { Number } \\
\end{array}$ & $\begin{array}{c}\text { Coordinate } \\
\text { Name }\end{array}$ & $\begin{array}{c}\text { Common Name } \\
\text { or Prefix }{ }^{a}\end{array}$ & Type $^{\text {b }}$ & $\begin{array}{c}l \\
(\mathrm{deg}) \\
\end{array}$ & $\begin{array}{c}b \\
(\mathrm{deg}) \\
\end{array}$ & $\begin{array}{l}\text { Max. Int. } \\
\text { (Crab) }\end{array}$ & $\begin{array}{l}P_{\text {orb }} \\
(\mathrm{hr}) \\
\end{array}$ & $\begin{array}{c}D \\
(\mathrm{kpc})\end{array}$ & $\begin{array}{c}\text { References } \\
\text { (distance) }\end{array}$ \\
\hline 1 & $1354-64$ & (GS) & LPT & 310.0 & -2.8 & 0.12 & 61.1 & $>25$ & 1 \\
\hline 2 & $1543-47$ & $(4 U)$ & LPT & 330.9 & +5.4 & 15 & 26.8 & $7.5 \pm 0.5$ & 2 \\
\hline 3 & $1550-564$ & (XTEJ) & LPT & 325.9 & -1.8 & 7.0 & 37.0 & $4.4 \pm 0.5$ & 3 \\
\hline 4 & $1655-40$ & (GROJ) & LPT & 345.0 & +2.5 & 3.9 & 62.9 & $3.2 \pm 0.5$ & 4 \\
\hline 5 & $1659-487$ & GX 339-4 & LPT & 338.9 & -4.3 & 1.1 & 42.1 & $9 \pm 3$ & 5 \\
\hline 6 & $1819.3-2525$ & V4641 Sgr & LPT & 6.8 & -4.8 & 13 & 67.6 & $9.9 \pm 2.4$ & 6 \\
\hline 7 & $1915+105$ & $(\mathrm{GRS})$ & LPT & 45.4 & -0.2 & 3.7 & 739 & $9 \pm 3$ & 7 \\
\hline 8 & $2023+338$ & $(\mathrm{GS})$ & LPT & 73.1 & -2.1 & 20 & 155.3 & $2.39 \pm 0.14$ & 8 \\
\hline 9 & $0422+32$ & (GROJ) & SPT & 166.0 & -12.0 & 3 & 5.1 & $2 \pm 1$ & 9,10 \\
\hline 10 & $0620-003$ & (A) & SPT & 210.0 & -6.5 & 50 & 7.8 & $1.06 \pm 0.12$ & 11 \\
\hline 11 & $1009-45$ & (GRS) & SPT & 275.9 & +9.4 & 0.8 & 6.8 & $3.82 \pm 0.27$ & 10 \\
\hline 12 & $1118+480$ & $(\mathrm{XTEJ})$ & SPT & 157.6 & +62.3 & 0.04 & 4.1 & $1.7 \pm 0.1$ & 12 \\
\hline 13 & $1124-683$ & Nova Mus 91 & SPT & 295.3 & -7.1 & 3 & 10.4 & $5.89 \pm 0.26$ & 10 \\
\hline 14 & $1650-500$ & $(\mathrm{XTEJ})$ & SPT & 336.7 & -3.4 & 0.6 & 7.7 & $2.6 \pm 0.7$ & 13 \\
\hline 15 & $1705-250$ & Nova Oph 77 & SPT & 358.2 & +9.1 & 3.6 & 12.5 & $8.6 \pm 2.1$ & 14 \\
\hline 16 & $1859+226$ & $(\mathrm{XTEJ})$ & SPT & 54.1 & +8.6 & 1.5 & $9.2^{\mathrm{c}}$ & $8 \pm 3$ & 10 \\
\hline 17 & $2000+251$ & $(\mathrm{GS})$ & SPT & 63.4 & -3.0 & 11 & 8.3 & $2.7 \pm 0.7$ & 14 \\
\hline 18 & $0020+593$ & IC $10 \mathrm{X}-1$ & PS & $\ldots$ & $\ldots$ & 0.00006 & 34.9 & $\ldots$ & $\ldots$ \\
\hline 19 & $0055-377$ & NGC 300-1 & PS & $\ldots$ & $\ldots$ & 0.00004 & 32.3 & $\ldots$ & $\ldots$ \\
\hline 20 & $0133+305$ & M33 X-7 & PS & $\ldots$ & $\ldots$ & 0.00002 & 82.9 & $\ldots$ & $\ldots$ \\
\hline 21 & $0538-641$ & LMC X-3 & PS & $\ldots$ & $\ldots$ & 0.06 & 40.9 & $\ldots$ & $\ldots$ \\
\hline 22 & $0540-697$ & LMC X-1 & PS & $\ldots$ & $\ldots$ & 0.03 & 93.8 & $\ldots$ & $\ldots$ \\
\hline 23 & $1956+350$ & Cyg X-1 & PS & $\ldots$ & $\ldots$ & 2.3 & 134.4 & $\ldots$ & $\ldots$ \\
\hline
\end{tabular}

Notes.

a The entries in parentheses are prefixes to the coordinate names that identify the discovery X-ray mission.

b PS: persistent source; LPT: long-period transient; SPT: short-period transient.

${ }^{c}$ Unconfirmed and uncertain value, see the text.

References. (1) Casares et al. 2009; (2) J. Orosz 2010, private communication; (3) Orosz et al. 2010; (4) Hjellming \& Rupen 1995; (5) Hynes et al. 2004; (6) Orosz et al. 2001; (7) Fender et al. 1999; (8) Miller-Jones et al. 2009; (9) Webb et al. 2000; (10) Hynes 2005; (11) Cantrell et al. 2010; (12) Gelino et al. 2006; (13) Homan et al. 2006; (14) Barret et al. 1996.

X-ray binaries, the mass function

$$
f(M) \equiv \frac{P_{\mathrm{orb}} K^{3}}{2 \pi G}=\frac{M \sin ^{3} i}{(1+q)^{2}}
$$

is the most important and secure observable. The orbital period $P_{\text {orb }}$ and the half-amplitude of the velocity curve of the secondary star $K$ can, in most cases, be determined precisely and accurately (Remillard \& McClintock 2006; Charles \& Coe 2006). These two quantities define the value of the mass function, which is an absolute lower limit on the mass of the compact object: i.e., $M \geqslant f(M)$. The mass function relates the black hole mass $M$, the orbital inclination angle $i$, and the mass ratio $q \equiv M_{2} / M$, where $M_{2}$ is the mass of the secondary star. Values of the mass function, the inclination, and the mass ratio for the 23 established black hole binaries are given in Table 2 . The mass-ratio estimates were obtained or derived from Orosz (2003) and Narayan \& McClintock (2005), with a few refinements based on the references cited in the table. Determining the black hole mass $M$, the quantity of interest, is challenging because in many cases it is difficult to obtain secure constraints on the inclination and the mass ratio.

Table 1 separates the 23 systems into three groups based on their X-ray behavior: the persistent sources, the long-period transients, and the short-period transients. This is important for understanding possible selection effects, which we explore in Section 4. Table 2, on the other hand, separates sources into three different groups based on the amount of data available on their mass ratios and inclinations. This impacts the inference of the masses of individual sources, which we discuss in Section 3. These tables sparely give a few key references for each source, which are supplemented by some additional references in the discussion that follows.

\subsubsection{The Persistent Sources}

These systems contain O/B-type secondaries and are persistently X-ray bright. Five of them have relatively massive black hole primaries, $M>10 M_{\odot}$. However, the mass of LMC X-3 is presently poorly constrained: $4 M_{\odot} \leqslant M \leqslant 11 M_{\odot}$ (Cowley 1992). In the case of Cyg X-1, a very wide range of masses down to $5 M_{\odot}$ has recently been considered (CaballeroNieves et al. 2009). However, recent VLBA observations have shown that the distance exceeds $1.5 \mathrm{kpc}$ (M. Reid 2010, private communication), which firmly establishes $M>8 M_{\odot}$. There are two caveats on the mass constraints on IC $10 \mathrm{X}-1$ and NGC 300-1: these results are based on less-reliable emissionline radial velocities and assume specific lower bounds on the masses of the secondary stars.

\subsubsection{The Long-period Transient Sources}

Nearly all transient black hole sources, which are fed by Roche-lobe overflow, exhibit long periods of deep quiescence during which the spectra of their secondary stars are prominent. For all eight of these long-period systems $\left(P_{\text {orb }}>1 \mathrm{~d}\right)$ there is strong evidence that the masses of their black hole primaries exceed $6 M_{\odot}$. We comment on the two weakest cases. (1) GX 339-4 has never reached a deep enough quiescent state 
Table 2

Dynamical Data for 23 Black Hole Binaries

\begin{tabular}{|c|c|c|c|c|c|c|c|}
\hline $\begin{array}{l}\text { Source } \\
\text { Number } \\
\end{array}$ & $\begin{array}{c}\text { Coordinate } \\
\text { Name }\end{array}$ & $\begin{array}{c}\text { Common Name } \\
\text { or Prefix }{ }^{\mathrm{a}}\end{array}$ & $\begin{array}{l}f(M) \\
\left(M_{\odot}\right) \\
\end{array}$ & $\begin{array}{c}q \\
q_{\min }-q_{\max } \\
\end{array}$ & $\begin{array}{c}i \\
(\operatorname{deg}) \\
\end{array}$ & $\begin{array}{c}M \\
\left(M_{\odot}\right) \\
\end{array}$ & References \\
\hline 1 & $0620-003$ & (A) & $2.76 \pm 0.01$ & $0.056-0.064$ & $51.0 \pm 0.9$ & $6.6 \pm 0.25$ & 1,2 \\
\hline 2 & $1543-47$ & $(4 \mathrm{U})$ & $0.25 \pm 0.01$ & $0.25-0.31$ & $20.7 \pm 1.5$ & $9.4 \pm 1.0$ & 3 \\
\hline 3 & $1550-564$ & (XTEJ) & $7.73 \pm 0.40$ & $0.0-0.040$ & $74.7 \pm 3.8$ & $9.1 \pm 0.6$ & 4 \\
\hline 4 & $1655-40$ & (GROJ) & $2.73 \pm 0.09$ & $0.37-0.42$ & $70.2 \pm 1.9$ & $6.3 \pm 0.27$ & 3,5 \\
\hline 5 & $1819.3-2525$ & V4641 Sgr & $3.13 \pm 0.13$ & $0.42-0.45$ & $75 \pm 2$ & $7.1 \pm 0.3$ & 3 \\
\hline 6 & $2023+338$ & $(\mathrm{GS})$ & $6.08 \pm 0.06$ & $0.056-0.063$ & $55 \pm 4$ & $12 \pm 2$ & 6 \\
\hline 7 & $0422+32$ & (GROJ) & $1.19 \pm 0.02$ & $0.076-0.31$ & $\ldots$ & $\ldots$ & 3,7 \\
\hline 8 & $1009-45$ & $(\mathrm{GRS})$ & $3.17 \pm 0.12$ & $0.12-0.16$ & $\ldots$ & $\ldots$ & 3,8 \\
\hline 9 & $1118+480$ & $(\mathrm{XTEJ})$ & $6.1 \pm 0.3$ & $0.035-0.044$ & $\ldots$ & $\ldots$ & $3,9,10$ \\
\hline 10 & $1124-683$ & Nova Mus 91 & $3.01 \pm 0.15$ & $0.11-0.21$ & $\ldots$ & $\ldots$ & 3,11 \\
\hline 11 & $1354-64$ & $(\mathrm{GS})$ & $5.73 \pm 0.29$ & $0.08-0.15$ & $\ldots$ & $\ldots$ & 12 \\
\hline 12 & $1650-500$ & (XTEJ) & $2.73 \pm 0.56$ & $0.0-0.2^{\mathrm{b}}$ & $\ldots$ & $\ldots$ & 13 \\
\hline 13 & $1659-487$ & GX 339-4 & $5.8 \pm 0.5$ & $0.0-0.4^{\mathrm{b}}$ & $\ldots$ & $\ldots$ & 14,15 \\
\hline 14 & $1705-250$ & Nova Oph 77 & $4.86 \pm 0.13$ & $0.0-0.053$ & $\ldots$ & $\ldots$ & 3,6 \\
\hline 15 & $2000+251$ & $(\mathrm{GS})$ & $5.01 \pm 0.12$ & $0.035-0.053$ & $\ldots$ & $\ldots$ & 3,6 \\
\hline 16 & $1915+105$ & (GRS) & $9.5 \pm 3.0$ & $0.025-0.091$ & $66 \pm 2$ & $\ldots$ & $16,17,18,19$ \\
\hline 17 & $1859+226$ & (XTEJ) & $7.4 \pm 1.1^{\mathrm{c}}$ & $\ldots$ & $\ldots$ & $\ldots$ & 20,21 \\
\hline 18 & $0020+593$ & IC $10 \mathrm{X}-1$ & $7.64 \pm 1.26$ & $\ldots$ & $\ldots$ & $>20$ & 22,23 \\
\hline 19 & $0055-377$ & NGC $300-1$ & $2.6 \pm 0.3$ & $\ldots$ & $\ldots$ & $>10$ & 24 \\
\hline 20 & $0133+305$ & M33 X-7 & $0.46 \pm 0.07$ & $\ldots$ & $\ldots$ & $15.65 \pm 1.45$ & 25 \\
\hline 21 & $0538-641$ & LMC X-3 & $2.3 \pm 0.3$ & $\ldots$ & $\ldots$ & $\ldots$ & 26 \\
\hline 22 & $0540-697$ & LMC X-1 & $0.886 \pm 0.037$ & $\ldots$ & $\ldots$ & $10.91 \pm 1.54$ & 27 \\
\hline 23 & $1956+350$ & Cyg X-1 & $0.251 \pm 0.007$ & $\ldots$ & $\ldots$ & $>8$ & 28,29 \\
\hline
\end{tabular}

Notes.

a The entries in parentheses are prefixes to the coordinate names that identify the discovery X-ray mission.

${ }^{\mathrm{b}}$ Estimated range based on extreme values observed for systems with comparable orbital periods.

${ }^{c}$ Unconfirmed and uncertain value, see the text.

References. (1) Neilsen et al. 2008; (2) Cantrell et al. 2010; (3) Orosz 2003; (4) Orosz et al. 2010; (5) Greene et al. 2001; (6) Charles \& Coe 2006; (7) Filippenko et al. 1995; (8) Filippenko et al. 1999; (9) McClintock et al. 2001; (10) Gelino et al. 2006; (11) Orosz et al. 1996; (12) Casares et al. 2009; (13) Orosz et al. 2004; (14) Hynes et al. 2003; (15) Muñoz-Darias et al. 2005; (16) Greiner et al. 2001; (17) Neil et al. 2007; (18) Harlaftis \& Greiner 2004; (19) Fender et al. 1999; (20) Filippenko \& Chornock 2001; (21) Zurita et al. 2002; (22) Prestwich et al. 2007; (23) Silverman \& Filippenko 2008; (24) Crowther et al. 2010; (25) Orosz et al. 2007; (26) Cowley 1992; (27) Orosz et al. 2009; (28) Caballero-Nieves et al. 2009; (29) M. Reid 2010, private communication.

to reveal its photospheric absorption lines, and its mass function was determined via the Bowen emission lines (Hynes et al. 2003). Furthermore, this system does not exhibit ellipsoidal light curves that allow its inclination to be constrained. For a defense of the $M=6 M_{\odot}$ lower limit see Muñoz-Darias et al. (2008). (2) GRS 1915+105: the pioneering mass measurement of this system by Greiner et al. (2001) requires confirmation. Their spectroscopic orbital period disagrees by $8 \%$ with a more recent photometric determination of the period (Neil et al. 2007). Again, there are no well-behaved ellipsoidal light curves that can be used to constrain the inclination, which in this case is inferred from a kinematic model of the relativistic radio jets (Mirabel \& Rodríguez 1999).

\subsubsection{The Short-period Transient Sources}

Significant constraints have been placed on the black hole primaries of only three of the nine short-period systems $\left(P_{\text {orb }}<\right.$ 1 day). It is difficult to obtain reliable inclination constraints for these systems because studies in quiescence of their small, latetype secondaries are compromised by the presence of a relatively strong and variable component of non-stellar light (e.g., Zurita et al. 2003; Cantrell et al. 2008), which is continually present in all of these systems.

A measurement of the inclination angle has been obtained only for A0620-00, the prototype system, which is 2 mag brighter than the other short period transients. The study by Cantrell et al. (2010), a tour de force that makes use of 32 photometric data sets spanning 30 years, relies on 10 data sets obtained when the source was in a "passive" quiescent state (Cantrell et al. 2008). As indicated in Table 2, the authors constrain the mass to be $M=6.6 \pm 0.25 M_{\odot}$. Meanwhile, the masses of three other systems (XTE J1118+480, Nova Oph 1977, and GS 2000+251) have by dint of their large mass functions been shown to exceed $6 M_{\odot}$ (Table 2). The large mass function of XTE J1859+226 (Table 2) suggests that it also should be included in this group; however, this result is unreliable, having only been presented in an IAU Circular (Filippenko \& Chornock 2001), and the orbital period is uncertain (Zurita et al. 2002). We exclude this source from further considerations on deriving the black hole mass distribution.

Among the 23 systems in Table 2, arguably the best candidate for hosting a low-mass black hole is GRO J0422+32. However, by briefly discussing recent attempts to determine the system's inclination and mass, we show that current results are presently unreliable: Beekman et al. (1997) constrained the inclination to lie in the range $i=10^{\circ}-31^{\circ}$ and concluded $M>9 M_{\odot}$. Webb et al. (2000) concluded $i<45^{\circ}$ and $M>2.2 M_{\odot}$ with a maximum mass that is not constrained. Gelino \& Harrison (2003) present the strongest evidence for a low mass; they conclude $i>43^{\circ}$ and $M<4.92 M_{\odot}$. However, in a more recent Keck $K$-band study Reynolds et al. (2007) found that "No 
Table 3

Thirty-two Transient Black Hole Candidates

\begin{tabular}{|c|c|c|c|c|c|c|c|}
\hline Source Number & Source Name & R.A. (J2000) & Decl. (J2000) & $l(\mathrm{deg})$ & $b(\operatorname{deg})$ & $I(\mathrm{Crab})^{\mathrm{a}}$ & References \\
\hline 1 & 1A $1524-61$ & 152817.2 & -615258 & 320.3 & -4.4 & 0.95 & 1 \\
\hline 2 & $4 U 1630-47$ & 163401.61 & -472334.8 & 336.9 & +0.3 & 1.4 & 1 \\
\hline 3 & XTE J1652-453 & 165220.33 & -45 2039.6 & 340.5 & -0.8 & 0.19 & 2 \\
\hline 4 & IGR J17098-3628 & 170945.93 & -362758.2 & 349.6 & +2.1 & 0.13 & 1 \\
\hline 5 & SAX J1711.6-3808 & 171137.1 & -380705.7 & 348.6 & +0.8 & 0.13 & 1 \\
\hline 6 & GRO J1719-24 & 171936.93 & -250103.4 & 359.9 & +7.0 & 1.5 & 1 \\
\hline 7 & XTE J1720-318 & 171958.994 & -314501.25 & 354.6 & +3.1 & 0.41 & 1 \\
\hline 8 & IGR J17269-4737 & 172649.28 & -473824.9 & 342.2 & -6.9 & 0.083 & 1 \\
\hline 9 & GRS $1730-312$ & 173352.3 & -311225 & 356.7 & +1.0 & 0.20 & 1 \\
\hline 10 & GRS 1737-31 & 174009 & -310224 & 357.3 & +0.6 & 0.026 & 1 \\
\hline 11 & GRS $1739-278$ & 174240.03 & -274452.7 & 0.7 & +1.2 & 0.86 & 1 \\
\hline 12 & KS J1739-304 & 174244.0 & -303051 & 358.3 & -0.3 & 0.009 & 1 \\
\hline 13 & 1E $1740.7-2942$ & 174354.83 & -294442.6 & 359.1 & -0.1 & 0.03 & 1 \\
\hline 14 & 1A $1742-289$ & 174537.0 & -290107 & 359.9 & -0.0 & 2.0 & 1 \\
\hline 15 & H1743-322 & 174615.57 & -321401.1 & 357.1 & -1.6 & 0.77 & 1 \\
\hline 16 & XTE J1748-288 & 174805.06 & -282825.8 & 0.7 & -0.2 & 0.64 & 1 \\
\hline 17 & IGR J17497-2821 & 174938.037 & -282117.37 & 1.0 & -0.5 & 0.03 & 1 \\
\hline 18 & SLX 1746-331 & 174948.3 & -331226 & 356.9 & -3.1 & 0.27 & 1 \\
\hline 19 & Swift J1752-223 & 175215.10 & -222032.78 & 6.4 & +2.1 & 0.11 & 3 \\
\hline 20 & Swift J1753.5-0127 & 175328.29 & -012706.22 & 24.9 & +12.2 & 0.16 & 4 \\
\hline 21 & XTE J1755-324 & 175528.6 & -322839 & 358.0 & -3.6 & 0.18 & 1 \\
\hline 22 & 4U 1755-33 & 175840.0 & -334827 & 357.2 & -4.9 & 0.10 & 1 \\
\hline 23 & GRS $1758-258$ & 180112.40 & -254436.1 & 4.5 & -1.4 & 0.02 & 1 \\
\hline 24 & SAX J1805.5-2031 & 180534 & -203048 & 9.6 & +0.3 & 0.051 & 1 \\
\hline 25 & XTE J1817-330 & 181743.54 & -330107.8 & 359.8 & -8.0 & 1.35 & 1 \\
\hline 26 & XTE J1818-245 & 181824.4 & -243218.0 & 7.4 & -4.2 & 0.51 & 1 \\
\hline 27 & Swift J1842.5-1124 & 184217.33 & -112500.6 & 21.7 & -3.2 & 0.04 & 5 \\
\hline 28 & EXO 1846-031 & 184639.8 & -030721 & 29.9 & -0.9 & 0.3 & 1 \\
\hline 29 & IGR J18539+0727 & 185354 & +0727 & 39.8 & +2.8 & 0.02 & 1 \\
\hline 30 & XTE J1856+053 & 185639 & +051948 & 38.3 & +1.3 & 0.07 & 1 \\
\hline 31 & XTE J1908+094 & 190853.077 & +092304.90 & 43.3 & +0.4 & 0.10 & 1 \\
\hline 32 & XTE J2012+381 & 201237.71 & +381101.1 & 75.4 & +2.2 & 0.16 & 1 \\
\hline
\end{tabular}

Notes. ${ }^{a}$ Approximate maximum X-ray intensity from the Liu et al. (2007) catalog supplemented in a few cases by data from other sources. In some instances, the intensity is for a higher energy band than the standard $\sim 2-10 \mathrm{keV}$ band.

References. (1) Liu et al. 2007; (2) Markwardt et al. 2009; (3) Torres et al. 2009; (4) Fender et al. 2005; (5) Krimm et al. 2008.

clear ellipsoidal modulation is present in the light curve...” and concluded "...that previous infrared-based attempts to constrain the mass of the putative black hole in this system are prone to considerable uncertainty." Thus, it appears that a far more comprehensive photometric study (cf. Cantrell et al. 2010) is required in order to obtain a firm mass constraint.

There are six systems in Table 2 with black holes of indeterminate mass. The prospects for measuring or usefully constraining the masses of two of them, LMC X-3 and XTE J1859-226, are bright: work on the former is almost complete (J. Orosz 2010, private communication), and the additional data required to confirm the orbital period and large mass function of the latter are obtainable. That leaves four systems (GRO J0422+32, GRS 1009-45, Nova Mus 1991, and XTE J1650-500) with black hole masses that are only very weakly constrained: $M>f(M) \gtrsim 1-3 M_{\odot}$. As indicated above and as Cantrell et al. (2010) have shown, it will be very challenging to place stronger and reliable constraints on the masses of these black holes.

\subsection{Thirty-two Transient Black Hole Candidates}

Data for $32 \mathrm{X}$-ray transient systems are given in Table 3. These systems lack radial velocity data and most even lack an optical counterpart. Thus, presently, there are no dynamical constraints on the masses of their compact primaries, which are believed to be black holes because they share certain characteristic X-ray properties with the 23 established black holes (McClintock \& Remillard 2006). As indicated in Table 3, the primary source of information about these systems is the catalog of Liu et al. (2007), and references therein. For additional information and references on many of these systems, see Table 4.3 and text in McClintock \& Remillard (2006).

\section{BLACK HOLE MASS MEASUREMENTS AND CONSTRAINTS}

In this section, we use the measurements of the mass functions, as well as any available constraints on the mass ratios and inclinations for the black holes in low-mass X-ray binaries shown in Table 2 in order to place quantitative constraints on the individual black hole masses. In particular, our aim is to derive the likelihood $P_{i}($ data $\mid M)$, which measures the chance of obtaining the particular set of data shown in Table 2 for the $i$ th source if that source had mass $M$.

We divide the sources into three categories based on the amount and quality of information regarding their mass ratios and inclinations:

1. For six sources, the mass ratios and the inclinations are tightly constrained, leading to well-determined black hole masses. In this case, the probability distribution can be 
described as a Gaussian

$$
P_{i}(\text { data } \mid M)=C_{i} \exp \left[\frac{-\left(M-M_{0, i}\right)^{2}}{2 \sigma_{M, i}^{2}}\right]
$$

with a mean $M_{0, i}$ and a standard deviation $\sigma_{M, i}$. In this and the following expressions, $C_{i}$ is a proper normalization constant such that

$$
\int_{0}^{\infty} P_{i}(\operatorname{data} \mid M) d M=1 .
$$

This category includes A0620-003, 4U 1543-47, XTE J1550-564, GRO J1655-40, V4641 Sgr, and GS $2023+338$.

2. For the sources in the second category, there is only a measurement of the mass function and constraints on the mass ratio $q$. Here, we assume a Gaussian probability distribution over the mass function with a mean $f_{0, i}$ and a standard deviation $\sigma_{0, i}$. For the mass ratio, we adopt a uniform distribution

$$
P(q) d q=\frac{d q}{q_{\max }-q_{\min }}
$$

between the minimum and maximum allowed mass ratios, $q_{\text {min }}$ and $q_{\text {max }}$, respectively. For each value of the mass ratio, the lack of eclipses implies a maximum value of the inclination, i.e., a minimum value of $\cos i$, such that

$$
(\cos i)_{\min }=0.462\left(\frac{q}{1+q}\right)^{1 / 3} .
$$

Assuming a uniform distribution over $\cos i$ subject to this constraint, i.e.,

$$
P(\cos i \mid q) d(\cos i)=\frac{d(\cos i)}{1-(\cos i)_{\min }}, \quad(\cos i)_{\min } \leqslant \cos i \leqslant 1,
$$

yields

$$
\begin{aligned}
P_{i}(\text { data } \mid M)= & C_{i} \int_{q_{\min }}^{q_{\max }} d q \int_{(\cos i)_{\min }}^{1} \frac{d(\cos i)}{1-(\cos i)_{\min }} \\
& \times \exp \left\{-\frac{\left[f_{0, i}-M \sin ^{3} i /(1+q)^{2}\right]^{2}}{2 \sigma_{f, i}^{2}}\right\} .
\end{aligned}
$$

The following nine sources belong to this category: GROJ 0422+32, GRS 1009-45, XTE J1118+480, Nova Mus 91, MS 1354-64, XTE J1650-500, GX 339-4, Nova Oph 77, and GS 2000+251.

3 . This last category includes only GRS $1915+105$, for which the mass function and the inclination have been measured, and the mass ratio has been constrained (see the discussion in Section 2 about the inclination measurement). In this case, we calculate the probability distribution over mass using Equation (7), supplemented by a Gaussian distribution over inclination

$$
\begin{aligned}
P_{i}(\text { data } \mid M)= & C_{i} \int_{q_{\min }}^{q_{\max }} d q \int_{(\cos i)_{\min }}^{1} \frac{d(\cos i)}{1-(\cos i)_{\min }} \\
& \times \exp \left\{-\frac{\left[f_{0, i}-M \sin ^{3} i /(1+q)^{2}\right]^{2}}{2 \sigma_{f, i}^{2}}-\frac{\left(i-i_{0}\right)^{2}}{2 \sigma_{i}^{2}}\right\} .
\end{aligned}
$$
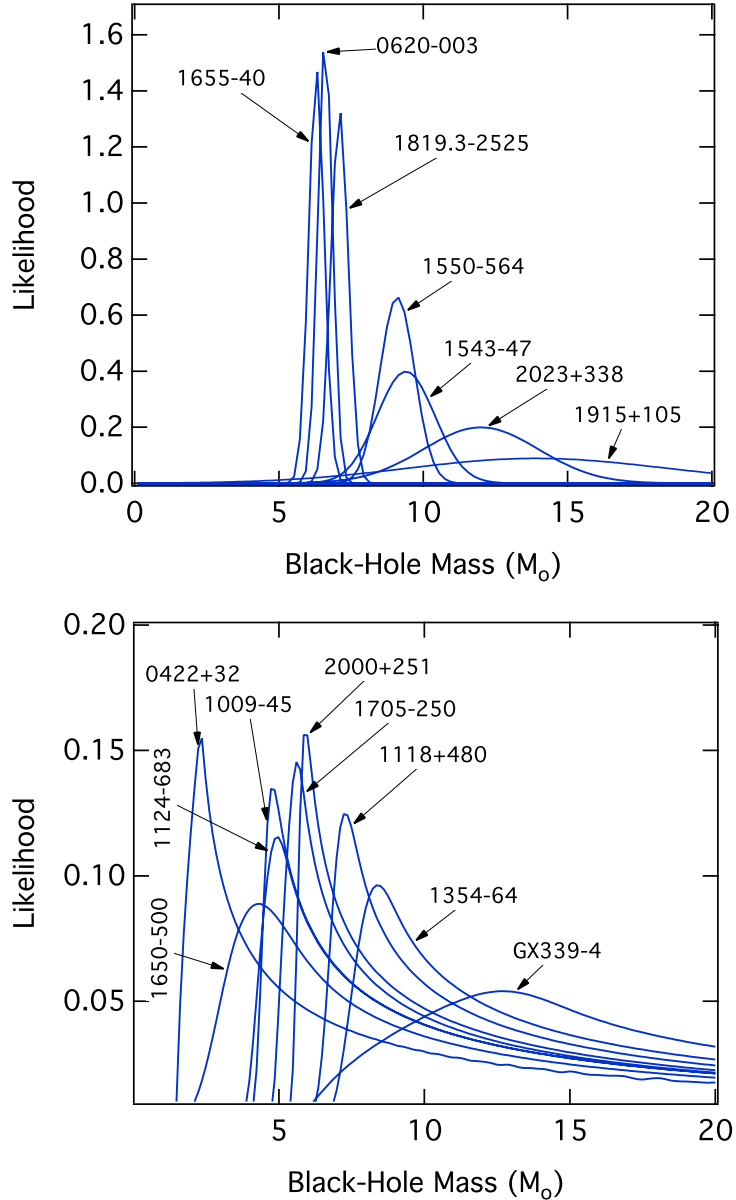

Figure 1. Likelihoods $P_{i}($ data $\mid M)$ for the 16 sources in low-mass X-ray binaries that have been securely identified as black holes. The top panel includes sources in categories (1) and (3), while the bottom panel shows those in category (2). The categories are based on the amount of information available on the mass ratios and inclinations of the black hole binaries and are discussed in more detail in the text.

(A color version of this figure is available in the online journal.)

Figure 1 shows the likelihoods $P_{i}($ data $\mid M)$ for the 16 sources in the above three categories. The top panel includes sources in categories (1) and (3), while the bottom panel shows those in category (2).

A clustering of the observed black hole masses between $\sim 6$ and $10 M_{\odot}$ is already evident from Figure 1 . In the next section, we will carry out a formal Bayesian analysis to determine the parameters of the underlying mass distribution that is consistent with the observed $P_{i}($ data $\mid M)$ shown here. If the likelihood for each source was narrow enough such that there was little or no overlap between them, then adding the likelihoods for the entire sample and coarsely binning the resulting distribution would provide a good estimate of the underlying mass distribution. Even though this condition is not entirely satisfied here, especially at the high-mass end, we nevertheless show in Figure 2 this approximate mass distribution to get a sense of its gross properties.

\section{THE INTRINSIC DISTRIBUTION OF BLACK HOLE MASSES}

In this section, we use a parametric form of the black hole mass distribution and the data discussed in Sections 2 and 3 in order to determine its parameters. We will first consider an 


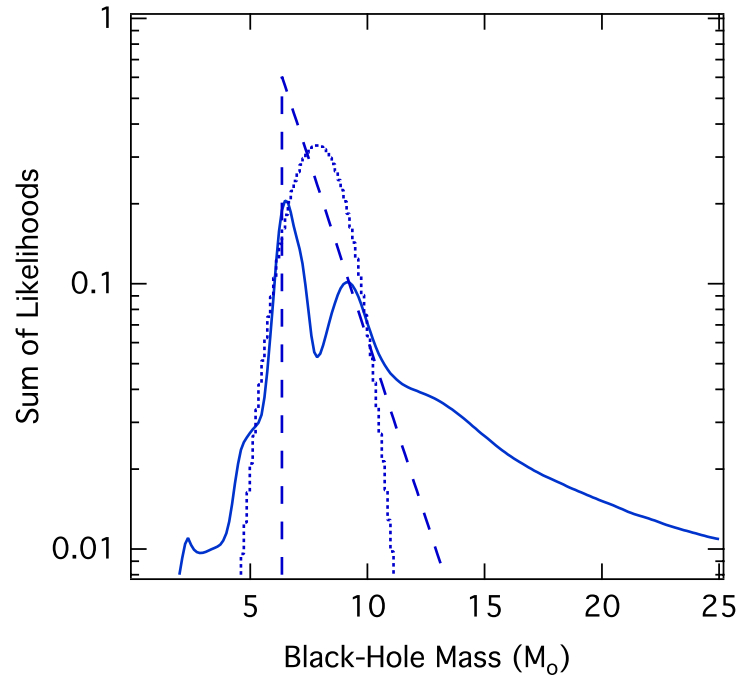

Figure 2. Solid line shows the sum of likelihoods for the mass measurements of the 16 black holes in low-mass X-ray binaries. Note that because of the highmass wings of the individual likelihoods, the shape of their sum is artificial at the high-mass end. The dashed and dotted lines show the exponential and Gaussian distributions, respectively, with parameters that best fit the data (see Section 4). (A color version of this figure is available in the online journal.)

exponentially decaying mass distribution with a cutoff given by

$$
\begin{aligned}
P\left(M ; M_{\text {scale }}, M_{\mathrm{c}}\right)= & \frac{\exp \left(M_{\mathrm{c}} / M_{\text {scale }}\right)}{M_{\text {scale }}} \\
& \times \begin{cases}\exp \left(-M / M_{\text {scale }}\right), & M>M_{\mathrm{c}} \\
0, & M \leqslant M_{\mathrm{c}}\end{cases}
\end{aligned}
$$

This choice of the mass distribution is motivated by theoretical expectations based on the energetics of supernova explosions, as well as the density profiles and mass distributions of presupernova stars. The typical value of the mass scale is expected to lie in the range $M_{\text {scale }} \sim 5.5-9 M_{\odot}$ (as we infer from the various figures in Fryer \& Kalogera 2001), whereas the cutoff mass is simply expected to be the maximum neutron-star mass. Our goal is to find the values of the mass scale $M_{\text {scale }}$ in the exponential and the cutoff mass $M_{\mathrm{c}}$ that maximize a properly defined likelihood and to estimate their uncertainties. We will show below that the particular choice of the functional form of the mass distribution does not affect the main conclusions of the paper.

In Section 3, we calculated, for each observed black hole, the probability $P_{i}($ data $\mid M)$, which measures the chance of making a particular observation if the black hole has mass $M$. What we want to calculate here is the probability $P\left(M_{\text {scale }}, M_{\mathrm{c}} \mid\right.$ data $)$, which measures the likelihood of the parameters of the black hole mass distribution, given the observations. Using Bayes' theorem, we can write this as

$$
P\left(M_{\text {scale }}, M_{\mathrm{c}} \mid \text { data }\right)=C_{2} P\left(\text { data } \mid M_{\text {scale }}, M_{\mathrm{c}}\right) P\left(M_{\text {scale }}\right) P\left(M_{\mathrm{c}}\right) \text {, }
$$

where $C_{2}$ is the normalization constant and $P\left(M_{\text {scale }}\right)$ and $P\left(M_{\mathrm{c}}\right)$ are the priors over the values of the mass scale and cutoff mass. We assume a flat prior over the mass scale between $M_{\text {scale }}=0$ and a maximum value $M_{\text {scale }}=M_{\max }$, i.e.,

$$
P\left(M_{\text {scale }}\right)= \begin{cases}0, & M_{\text {scale }} \leqslant 0 \\ \frac{1}{M_{\max }}, & 0<M_{\text {scale }} \leqslant M_{\max } \\ 0, & M_{\text {scale }}>M_{\max }\end{cases}
$$

The upper limit $M_{\max }$ is imposed mostly for computational reasons and does not affect the results. We also adopt a similar

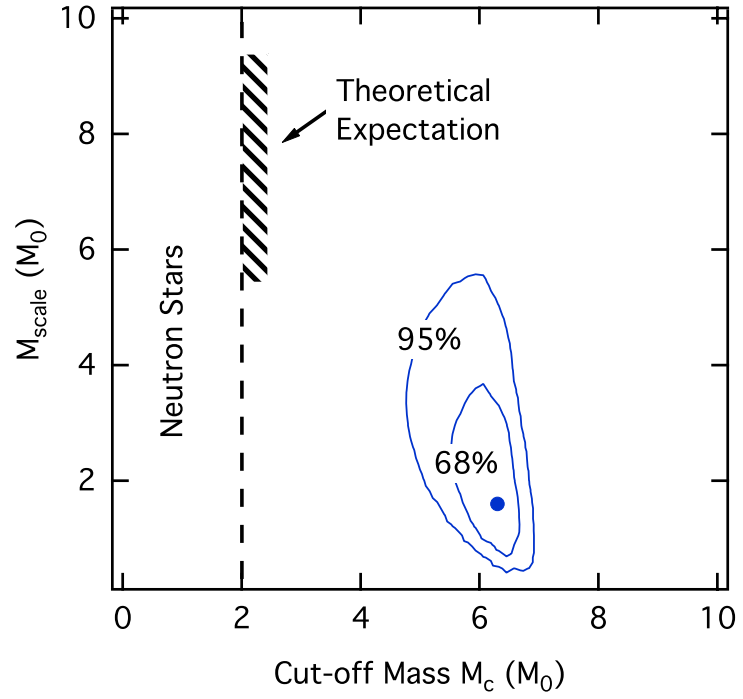

Figure 3. Parameters of an exponential black hole mass distribution with a lowmass cutoff. The cutoff mass is well above theoretical expectations, indicating a sizable gap between neutron-star and black hole masses. Furthermore, the mass scale in the exponential is significantly smaller than theoretical expectations.

(A color version of this figure is available in the online journal.)

prior over the cutoff mass between the maximum neutron-star mass, which we set to $2 M_{\odot}$, and the minimum well-established mass measurement for a black hole. As will be evident from the results, the particular choice of this range does not affect the measured parameters. We also repeated this analysis with logarithmic priors in the two parameters and found that the results are insensitive to the choice of priors.

In Equation (10), the quantity $P\left(\right.$ data $\left.\mid M_{\text {scale }}, M_{\mathrm{c}}\right)$ measures the chance that we make a particular set of observations for the ensemble of black holes, given the values of the parameters of the mass distribution. We need now to estimate this quantity, given the likelihoods for the individual sources. We will assume that each measurement is independent of all the others, so that

$$
P\left(\text { data } \mid M_{\text {scale }}, M_{\mathrm{c}}\right)=\prod_{i} \int d M P_{i}(\text { data } \mid M) P\left(M ; M_{\text {scale }}, M_{\mathrm{c}}\right) .
$$

Combining this last equation with Equation (10) we obtain

$$
\begin{aligned}
P\left(M_{\text {scale }}, M_{\mathrm{c}} \mid \text { data }\right)= & C P\left(M_{\text {scale }}\right) P\left(M_{\mathrm{c}}\right) \prod_{i} \\
& \times \int d M P_{i}(\text { data } \mid M) P\left(M ; M_{\text {scale }}, M_{\mathrm{c}}\right),
\end{aligned}
$$

where $C$ is the overall normalization constant.

We show in Figure 3 the $68 \%$ and $95 \%$ confidence contours of the mass scale and cutoff mass that best describe the observations and compare them to the theoretical expectation. The lack of black holes below $\sim 5 M_{\odot}$ and the rapid decline of the exponential distribution at the high-mass end are both remarkable (see the dashed line in Figure 2). The latter result is not at odds with the relatively high mass of GRS $1915+105$ because of the wide and shallow mass probability distribution of this source.

In order to explore whether the small number of sources with very well-determined masses dominate this result, we did the following test. We repeated the calculation using the mass functions and constraints on the mass ratios for all sources 


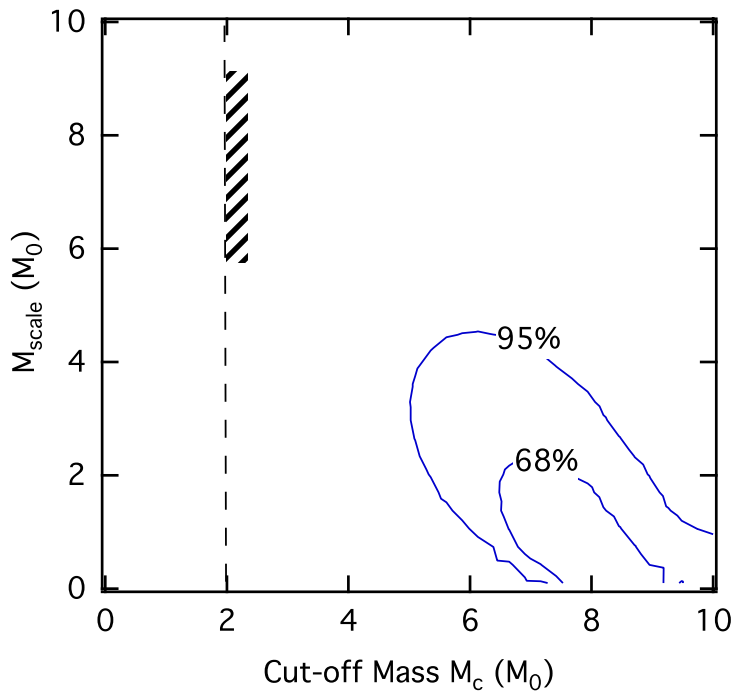

Figure 4. Parameters of an exponential black hole mass distribution when all possible inclinations for each binary system were allowed (i.e., no inclination information was taken into account). The existence of the low-mass gap and the small exponential mass scale remains robust.

(A color version of this figure is available in the online journal.)

but ignoring any information on the inclinations of the binary systems. This, in effect, is equivalent to treating all sources in category (1) using the formalism we applied to sources in category (2), integrating over all possible values of inclination. Figure 4 shows the $68 \%$ and $95 \%$ confidence contours of the parameters of the exponential distribution in this test case. Although the allowed range of values is increased, as expected, the low-mass gap and the discrepancy with the theoretically expected mass scale remain robust.

The narrowness of the mass distribution implied by the above results motivated us to explore different functional forms of the underlying distribution, and in particular, a Gaussian function. The Gaussian function here serves as a phenomenological two-parameter description of a narrow distribution and is not necessarily motivated by theory. We show in Figure 5 the parameters of such a Gaussian distribution that best describes the observations. The masses of all 16 black holes are consistent with a narrow distribution at $7.8 \pm 1.2 M_{\odot}$. This result is in agreement with an earlier, more limited, study by Bailyn et al. (1998).

\section{OBSERVATIONAL SELECTION EFFECTS}

In the previous section, we discussed two distinct results we obtained on the mass distribution of stellar black holes using the data on 16 sources in low-mass X-ray binaries: the cutoff mass at the low end is $\gtrsim 5 M_{\odot}(95 \%$ confidence $)$, indicating a significant lack of black holes in the $\sim 2-5 M_{\odot}$ range, and the mass scale in the exponential is $\sim 1.2 M_{\odot}$, indicating a rapid decline of the distribution at the high-mass end. In other words, the black hole mass distribution is narrow and can also be represented with a Gaussian at $7.8 \pm 1.2 M_{\odot}$. We now explore two questions about our results: (1) whether any observational selection effects could have biased the black hole mass distribution we inferred for transient sources and (2) whether the black holes in transient low-mass X-ray binaries are a representative sample of the population of stellar black holes in the Galaxy.

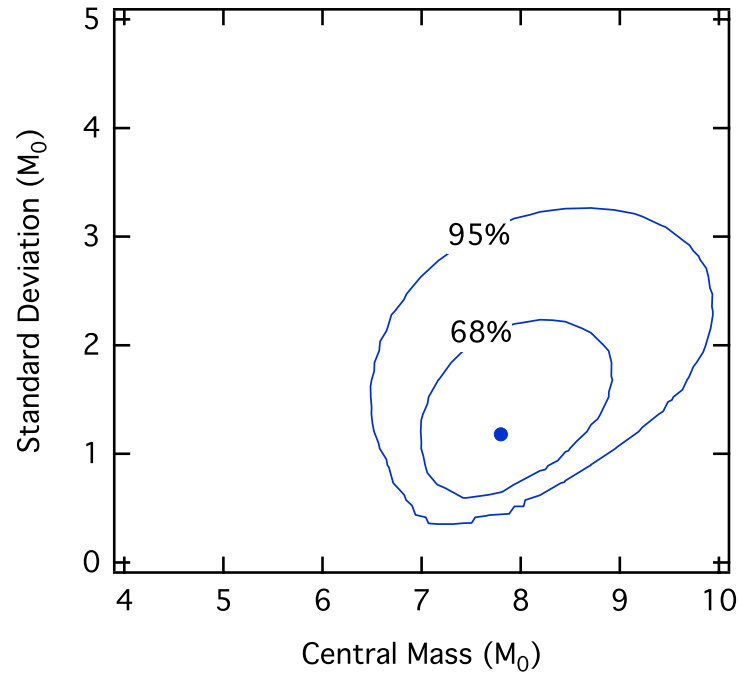

Figure 5. Parameters of a Gaussian black hole mass distribution. The data are best described by a narrow distribution at $7.8 \pm 1.2 M_{\odot}$.

(A color version of this figure is available in the online journal.)

\subsection{Selection Biases in the Mass Distribution of Black Holes in Transient $X$-ray Binaries}

The masses of stellar black holes have generally been measured when the black holes (1) reside in binary systems, (2) are transient X-ray sources, and (3) reach peak fluxes above $1 \mathrm{Crab}$ (about $2.5 \times 10^{-8} \mathrm{erg} \mathrm{s}^{-1} \mathrm{~cm}^{-2}$ ) during their outbursts. The first requirement naturally arises from the nature of the dynamical mass measurements. The second requirement ensures that optical observations of the companion star can be carried out during the quiescent phase so that the radial velocity measurements are not contaminated by the emission from the accretion disk. ${ }^{4}$ Finally, the third requirement is a common strategy of observing campaigns that aims to select among the transients those sources that are the closest and the least obscured.

Black hole transients have been observed in the last four decades with a large collection of X-ray telescopes. We, therefore, tried to verify and quantify the third requirement for followup by compiling the highest flux level observed from each transient black hole or black hole candidate in outburst by any instrument (see Table 1). We show in Figure 6 the histogram of the highest flux levels observed during outburst from the 16 confirmed black holes (in red) and from the black hole candidates (in blue). The dichotomy between the fluxes of the two groups is striking and indeed shows that primarily the brightest sources have been followed up for dynamical mass measurements.

We then explored whether the $1 \mathrm{Crab}$ flux threshold during outburst could lead to a selection bias against low-mass black holes. Setting a flux limit for optical follow-ups restricts, in principle, the volume of the Galaxy that is sampled. If, in addition, the low-mass black holes form an intrinsically fainter population than that of more massive black holes, this could indeed introduce a selection bias.

Consider, for example, the case in which the maximum luminosity during the outburst of an X-ray transient is proportional to the mass of the black hole, so that the binary systems with low-mass black holes are intrinsically dimmer than their high mass counterparts. Because the volume of the disk component of the Galaxy scales as the distance squared, the volume sam-

\footnotetext{
4 The dynamical Bowen technique (Steeghs \& Casares 2002) offers the possibility of measuring the masses of black holes in persistent systems.
} 


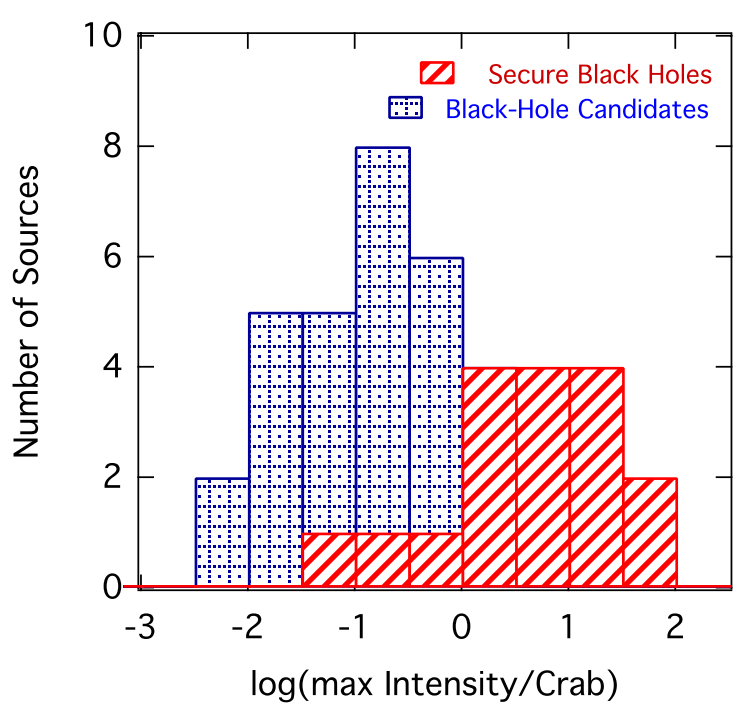

Figure 6. Histogram of maximum flux observed during the outbursts of black holes with dynamical mass determinations (red) and other black hole candidates (blue). A clear dichotomy between the two groups at $1 \mathrm{Crab}$ is evident. (A color version of this figure is available in the online journal.)

pled by sources selected for follow-up observations scales as the mass of the black hole. If the mass distribution of black holes was rather flat, then, on average, $\sim 4$ times more $\sim 8 M_{\odot}$ black holes would be followed up than $\sim 2 M_{\odot}$ black holes, leading to an artificial reduction in the number of low-mass black holes.

If there were indeed a population of intrinsically fainter, lowmass black holes, for the 16 more massive ones that have been observed, four low-mass black holes should have been found within the same flux limits according to the previous argument. In contrast, there is none in the current sample. This fact argues that the lack of low-mass black holes is real.

Another way to determine whether the volume-limited sample introduces a mass bias is to compare the number and distribution in the Galaxy of secure black hole sources to those of the black hole candidates. We plot in Figure 7 the galactic distribution of the black holes with secure mass determinations. It is evident that these sources lie within $\simeq 1 / 3$ of the volume of the galactic disk. As a result, if the observed black holes are a representative sample of the true population, our catalog of black hole candidates should contain twice as many sources that are fainter simply because they lie further away in the Galaxy compared to the 16 sources that have been followed up. This is precisely the ratio of the number of black hole candidate sources in Table 3 to that of secure black holes in Table 1: 32:16.

The 16 secure black holes have a median intensity of $3.6 \mathrm{Crab}$ and a median distance of $\sim 4 \mathrm{kpc}$. The 32 black hole candidates, on the other hand, have a median intensity of $0.18 \mathrm{Crab}$, so we expect them to be on average $\sqrt{3.6 / 0.18}=4.5$ times farther. (Note, however, that confirmed black holes have benefited from almost continuous monitoring that led to an accurate measurement of the maximum intensities. The black hole candidates have been observed more sparsely, so that their maximum reported intensity is in most cases likely to be below the actual maximum intensity that the sources reached). This further supports the idea that the black hole candidates lie in the far two-thirds of the Galaxy.

We, therefore, conclude that there is no room within the 32 black hole candidates to hide any sizable population of sources that are fainter because they are intrinsically less massive. The fact that our sample of secure black holes is flux limited does

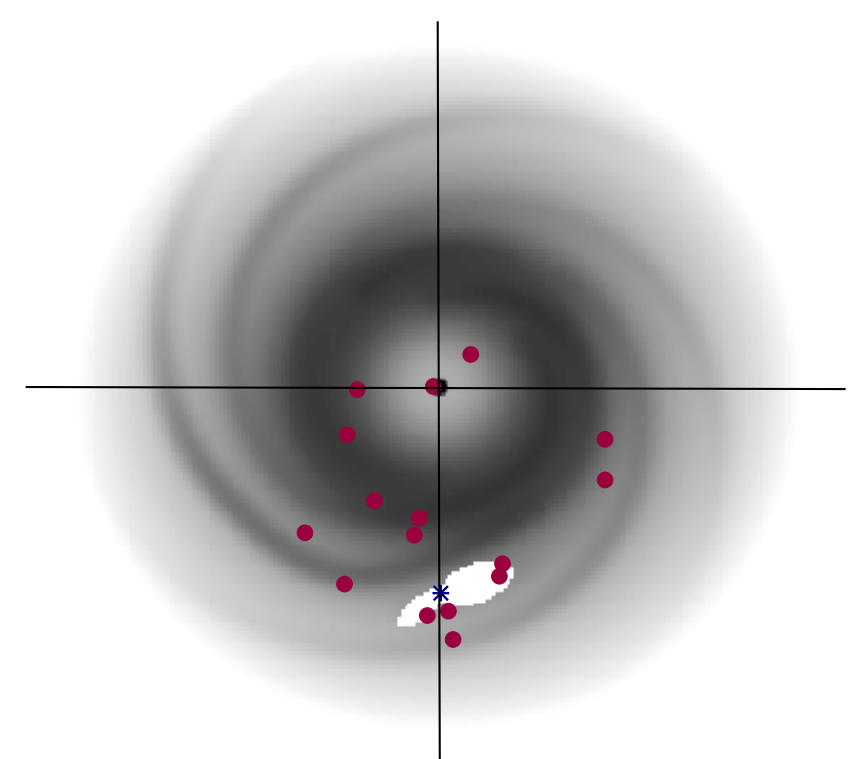

Figure 7. Positions on the Galactic disk of the black holes with secure dynamical mass measurements. The background grayscale image is the electron distribution in the Galaxy according to the model of Cordes \& Lazio (2002). The plus sign represents the position of the Sun.

(A color version of this figure is available in the online journal.)

not imply that it is luminosity limited, and, therefore, does not introduce any selection bias against the detection of low-mass black holes.

\subsection{Are Black Holes in Transient Low-mass X-ray Binaries a Representative Sample?}

The sample of 16 sources that we used to infer the mass distribution of stellar black holes has been drawn from transient low-mass X-ray binaries. ${ }^{5}$ Here, we consider the possibility that low-mass black holes exist, but not in transient low-mass X-ray binaries. In turn, we will consider the possibility that low-mass black holes are primarily in persistent binaries or that they are not members of binary systems at all.

1. Are accreting low-mass $\left(2-5 M_{\odot}\right)$ or high-mass $\left(\gtrsim 9 M_{\odot}\right)$ black holes persistent X-ray sources? Most known neutronstar low-mass X-ray binaries are persistent sources, whereas the majority of black hole binaries are transient systems. (The latter statement is primarily a consequence of the fact that the transient behavior has so far been required in establishing the black hole nature of a compact object in a majority of the sources). Is it possible that low-mass black holes behave more like neutron stars than their more massive counterparts?

The most prominent explanation of the transient behavior of X-ray binaries is the irradiated disk instability model (van Paradijs 1996; King et al. 1996; Dubus et al. 1999). In this model, whether an X-ray binary is a transient depends only weakly on the nature of the primary but is mainly determined by the orbital period of the system, the mass of the primary, and the evolutionary state of the companion star (King et al. 1996). Thus, the expectation is that a $4 M_{\odot}$ black hole binary should behave like one containing a $6 M_{\odot}$ black hole if they have comparable orbital periods. Furthermore, our sample of 16 transient black hole sources includes both short-period and long-period systems, indicating that the

\footnotetext{
5 Persistent sources with O/B-type secondaries have also been successfully targeted for mass measurements; see Tables 1 and 2.
} 
transient behavior is seen over a range of orbital periods. We, therefore, find it unlikely, albeit still possible, that lowmass black holes would preferentially exist in persistent binaries if the distribution of their orbital periods is similar to that of the confirmed black holes.

We, nevertheless, explore whether the known number of persistent X-ray sources in the Galaxy is large enough to harbor the low-mass back holes if, indeed, all of the latter are persistent systems. To this end, we analyzed the catalog of low-mass X-ray binaries by Liu et al. (2007). Starting with the total number of non-pulsing X-ray binaries in the Galaxy, we identified the following categories: (1) confirmed black holes, which refer to those with dynamical mass measurements; (2) black hole candidates, which are sources with no mass determinations, but possess spectra and radio properties that resemble those of the confirmed black holes; (3) unidentified sources, which are those with significant number of observations but no distinguishing characteristics to reveal the nature of the primary; (4) neutron stars, which are sources that exhibit thermonuclear $\mathrm{X}$-ray bursts; and (5) sources for which there is very little information. In Table 4, we show the numbers and relative fractions of sources in these categories.

Table 4 indicates that there are a total of 72 known persistent X-ray sources. Forty-six out of these 72 sources have shown thermonuclear bursts and can securely be identified as neutron stars, leaving at most 26 sources as possible low-mass black hole binaries. In reality, only two of these remaining 26 sources show evidence for black hole nature, and a further seven show no thermonuclear bursts even though they have been extensively observed. We will, nevertheless, allow the possibility that all can harbor lowmass black holes.

For any integrable (i.e., declining faster than logarithmic) distribution of black hole masses that does not have a low-mass gap, there should be more black holes with masses in the $2-5 M_{\odot}$ range than with masses $\geqslant 5 M_{\odot}$. If all the 26 persistent sources were low-mass black holes, we would expect this number to be larger than the total number of transient (higher mass) black hole sources in the Galaxy. From the galactic distribution of transient black hole binaries, we estimated (see previous subsection) that there are at least three times as many black holes in the Galaxy with masses $\geqslant 5 M_{\odot}$ than our current sample of 16. Taking into account the short duty cycles of transient black hole binaries, we can safely infer that the total number of such systems in the galaxy is $\gg 48$, which is significantly larger than 26 . As a result, even if all the 26 non-bursting sources were low-mass black holes, they would not be enough to efficiently close the low-mass gap between $\sim 2$ and $\sim 5 M_{\odot}$ in the inferred distribution. Nevertheless, applying the dynamical Bowen technique to persistent low-mass X-ray binaries will help explore further this possibility.

2. Are low-mass $\left(2-5 M_{\odot}\right)$ or high-mass $\left(\gtrsim 9 M_{\odot}\right)$ black holes not members of X-ray binary systems? The fraction of binaries that survive the supernova explosion is determined by a number of factors, such as the orbital separation, the amount of mass loss, and the kick velocity during the supernova explosion. These factors depend on the progenitor and the remnant masses and may work in a way to hinder the formation of low-mass X-ray binaries with low- or high-mass black holes. Moreover, even if the
Table 4

Population of Low-mass X-ray Binaries in the Galaxy

\begin{tabular}{lccc}
\hline \hline \multicolumn{1}{c}{ Primary } & Type & Number & Fraction \\
\hline Neutron star & Persistent & 46 & $28 \%$ \\
Neutron star & Transient & 39 & $23 \%$ \\
Confirmed BH & Persistent & 0 & $0 \%$ \\
Confirmed BH & Transient & 16 & $9 \%$ \\
BH candidate & Persistent & 2 & $1 \%$ \\
BH candidate & Transient & 30 & $18 \%$ \\
Unidentified & Persistent & 7 & $4 \%$ \\
Unidentified & Transient & 3 & $2 \%$ \\
Little information & Persistent & 17 & $11 \%$ \\
Little information & Transient & 7 & $4 \%$ \\
\hline
\end{tabular}

binaries survive the explosions, systems with low- or highmass remnants may become wider and perhaps never reach contact. It appears unlikely that this is the explanation of the paucity of low-mass black holes between the systems with dynamical mass measurements (see Fryer \& Kalogera 2001), although addressing this question would only be possible through detailed population synthesis calculations.

On the other hand, the particular evolutionary path that leads to the formation of black hole X-ray binaries might be responsible for the lack of systems with relatively massive black holes $\left(\gtrsim 9 M_{\odot}\right)$. Mass transfer in the low-mass X-ray binary phase requires small orbital separations between the black hole and the low-mass companion. The common envelope phase, which is invoked to reduce sufficiently the orbital separation of the binary before the supernova, is thought to lead to the expulsion of the hydrogen envelope of the pre-supernova star, leaving behind a bare helium core. Furthermore, the winds from the resulting helium cores in a Wolf-Rayet phase are expected to lead to further mass loss, albeit at rates that are highly uncertain. As a result, it is possible that the black hole masses in contact binaries are capped at $\simeq 10 M_{\odot}$, although this value depends strongly on the assumed mass-loss rates (Woosley et al. 2002). This evolutionary path could provide a natural explanation for the rapid decline of the inferred mass function at the high-mass end. The same mechanism does not necessarily cap the mass of the black holes in high-mass X-ray binaries, which are wind-fed.

\section{IMPLICATIONS FOR BLACK HOLE FORMATION}

We now explore the implications of the narrow mass distribution of black holes in low-mass X-ray binaries for the progenitors of these systems and the supernovae that form the black holes.

As we discussed in the previous section, the rapid decline at the high-mass end of the inferred distribution is plausibly the result of the particular binary formation channel that leads to low-mass X-ray binaries. In contrast to this population, relatively more massive black holes $\left(>20 M_{\odot}\right)$ have been detected in high-mass X-ray binaries (see the persistent sources in Table 2). This argues that there is no intrinsic barrier for the formation of massive black holes as the end stages of stellar evolution but that the differences between the black hole masses in the two binary populations are due to the different evolutionary paths that they follow. Observational studies of mass loss from stars with hydrogen and helium envelopes, in isolation and in tight binary systems, will elucidate and test these ideas.

The low-mass gap, on the other hand, cannot be accounted for by observational selection effects (even though the mass measurement method requires a particular choice of targets and 
the observing strategy has resulted in a flux-limited sample). It is also unlikely that it results from the particular evolutionary paths of low-mass X-ray binaries. Therefore, it appears to be real.

The observed distribution of masses could be different than the distribution of birth masses because mass transfer in the $\mathrm{X}$-ray binary phase could have caused the black holes to evolve away from the masses they were born with. Population synthesis models (e.g., Fragos et al. 2010) show, however, that the least massive black holes are preferentially born with lowmass companions $\left(\lesssim 2 M_{\odot}\right)$ and, therefore, could not accrete a significant amount of matter over the lifetime of the binary. When the companions are more massive than $5 M_{\odot}$ at the start of the mass transfer phase, the black holes are born with masses that are also $\gtrsim 5 M_{\odot}$. As a result, the accretion in the binary phase is not likely to bridge the gap between 2 and $5 M_{\odot}$.

It has been suggested that black hole evaporation in braneworld gravity models can lead to a deficiency of low-mass black holes in the steady-state population. A gap could be created in this context because the rate of evaporation in braneworld gravity is very rapid and increases with decreasing black hole mass (Postnov \& Cherepashchuk 2003). However, recent constraints on the rate of evaporation obtained using the current population of black holes preclude this possibility (Johannsen et al. 2009).

The paucity of black holes with masses less than $5 M_{\odot}(95 \%$ confidence) are likely, therefore, to be related to the physics of supernova explosions that lead to the formation of black holes. Numerical simulations of supernova explosions typically generate a continuous distribution of black hole masses that decays as an exponential (Fryer 1999; Fryer \& Kalogera 2001). The continuity of masses is primarily a consequence of the relatively gradual dependence of explosion energies on the masses of the progenitors and the fact that explosion energies are still comparable to (although smaller than) the binding energy of the stellar envelopes. Although creating a mass gap is difficult given the current understanding of the supernova energetics, it has been suggested that it can be achieved under the ad hoc assumption that the explosion energy has a step-function dependence on progenitor mass and that it plunges to zero for stars more massive than $\sim 25 M_{\odot}$ (Fryer \& Kalogera 2001).

Such a bimodality in the energies of explosions that form neutron stars versus black holes should become apparent in the large sample of supernovae anticipated from the ongoing surveys that are sensitive to underluminous core-collapse supernovae.

We thank Chris Fryer for stimulating discussions and Ron Remillard for sharing his private catalog of black hole candidates. F.Ö. and D.P. thank the ITC at the Harvard-Smithsonian Center for Astrophysics for their hospitality. F.Ö. acknowledges support from NSF grant AST 07-08640 and Chandra Theory grant TMO-11003X. D.P. was supported by the NSF CAREER award NSF 0746549.

\section{REFERENCES}

Abadie, et al. (LIGO Scientific Collaboration) 2010, Class. Quantum Grav., 27, 173001

Bailyn, C. D., Jain, R. K., Coppi, P., \& Orosz, J. A. 1998, ApJ, 499, 367

Barret, D., McClintock, J. E., \& Grindlay, J. E. 1996, ApJ, 473, 963

Beekman, G., et al. 1997, MNRAS, 290, 303

Caballero-Nieves, S. M., et al. 2009, ApJ, 701, 1895

Cantrell, A. G., Bailyn, C. D., McClintock, J. E., \& Orosz, J. A. 2008, ApJ, 673, L159

Cantrell, A. G., et al. 2010, ApJ, 710, 1127
Casares, J., et al. 2009, ApJS, 181, 238

Charles, P. A., \& Coe, M. J. 2006, in Compact Stellar X-ray Sources, ed. W. Lewin \& M. van der Klis (Cambridge: Cambridge Univ. Press)

Cordes, J. M., \& Lazio, T. J. W. 2002, arXiv:astro-ph/0207156

Cowley, A. P. 1992, ARA\&A, 30, 287

Crowther, P. A., et al. 2010, MNRAS, 403, L41

Dubus, G., Lasota, J.-P., Hameury, J.-M., \& Charles, P. 1999, MNRAS, 303, 139

Fender, R., Garrington, S., \& Muxlow, T. 2005, ATel, 558

Fender, R. P., et al. 1999, MNRAS, 304, 865

Filippenko, A. V., \& Chornock, R. 2001, IAU Circ., 7644, 2

Filippenko, A. V., Matheson, T., \& Ho, L. C. 1995, ApJ, 455, 614

Filippenko, A. V., et al. 1999, PASP, 111, 969

Fragos, T., Tremmel, M., Rantsiou, E., \& Belczynski, K. 2010, ApJ, 719, L79

Fryer, C. L. 1999, ApJ, 522, 413

Fryer, C. L., Heger, A., Langer, N., \& Wellstein, S. 2002, ApJ, 578, 335

Fryer, C. L., \& Kalogera, V. 2001, ApJ, 554, 548

Gelino, D. M., \& Harrison, T. E. 2003, ApJ, 599, 1254

Gelino, D. M., et al. 2006, ApJ, 642, 438

Greene, J., Bailyn, C. D., \& Orosz, J. A. 2001, ApJ, 554, 1290

Greiner, J., Cuby, J. G., \& McCaughrean, M. J. 2001, Nature, 414, 522

Harlaftis, E. T., \& Greiner, J. 2004, A\&A, 414, L13

Hjellming, R. M., \& Rupen, M. P. 1995, Nature, 375, 464

Homan, J., et al. 2006, MNRAS, 366, 235

Hynes, R. I. 2005, ApJ, 623, 1026

Hynes, R. I., Steeghs, D., Casares, J., Charles, P. A., \& O’Brien, K. 2003, ApJ, 583, L95

Hynes, R. I., Steeghs, D., Casares, J., Charles, P. A., \& O’Brien, K. 2004, ApJ, 609,317

Johannsen, T., Psaltis, D., \& McClintock, J. E. 2009, ApJ, 691, 997

King, A. R., Kolb, U., \& Burderi, L. 1996, ApJ, 464, L127

Krimm, H. A., et al. 2008, ATel, 1610

Liu, Q. Z., van Paradijs, J., \& van den Heuvel, E. P. J. 2007, A\&A, 469, 807

Markwardt, C. B., Beardmore, A. P., Miller, J., \& Swank, J. H. 2009, ATel, 2120

McClintock, J. E., \& Remillard, R. A. 2006, in Compact Stellar X-ray Sources, ed. W. Lewin \& M. van der Klis (Cambridge: Cambridge Univ. Press), 157

McClintock, J. E., et al. 2001, ApJ, 551, L147

Miller-Jones, J. C. A., et al. 2009, ApJ, 706, L230

Mirabel, I. F., \& Rodríguez, L. F. 1999, ARA\&A, 37, 409

Muñoz-Darias, T., Casares, J., \& Martínez-Pais, I. G. 2005, ApJ, 635, 502

Muñoz-Darias, T., Casares, J., \& Martínez-Pais, I. G. 2008, MNRAS, 385, 2205

Narayan, R., \& McClintock, J. E. 2005, ApJ, 623, 1017

Narayan, R., Piran, T., \& Shemi, A. 1991, ApJ, 379, L17

Neil, E. T., Bailyn, C. D., \& Cobb, B. E. 2007, ApJ, 657, 409

Neilsen, J., Steeghs, D., \& Vrtilek, S. D. 2008, MNRAS, 384, 849

Orosz, J. A. 2003, in IAU Symp. 212, A Massive Star Odyssey: From Main Sequence to Supernova, ed. K. van der Hucht et al. (San Francisco, CA: ASP), 365

Orosz, J. A., Bailyn, C. D., McClintock, J. E., \& Remillard, R. A. 1996, ApJ, 468,380

Orosz, J. A., McClintock, J. E., Remillard, R. A., \& Corbel, S. 2004, ApJ, 616, 376

Orosz, J. A., Steiner, J. F., McClintock, J. E., Torres, M. A. P., Remillard, R. A., \& Bailyn, C. D. 2010, ApJ, submitted

Orosz, J. A., et al. 2001, ApJ, 555, 489

Orosz, J. A., et al. 2007, Nature, 449, 872

Orosz, J. A., et al. 2009, ApJ, 697, 573

Postnov, K. A., \& Cherepashchuk, A. M. 2003, Astron. Rep., 47, 989

Prestwich, A. H., et al. 2007, ApJ, 669, L21

Rau, A., et al. 2009, PASP, 121, 1334

Remillard, R. A., \& McClintock, J. E. 2006, ARA\&A, 44, 49

Reynolds, M. T., Callanan, P. J., \& Filippenko, A. V. 2007, MNRAS, 374, 657

Silverman, J. M., \& Filippenko, A. V. 2008, ApJ, 678, 17L

Smartt, S. J. 2009, ARA\&A, 47, 63

Steeghs, D., \& Casares, J. 2002, ApJ, 568, 273

Timmes, F. X., Woosley, S. E., \& Weaver, T. A. 1996, ApJ, 457, 834

Torres, M. A. P., Jonker, P. G., Steeghs, D., Yan, H., Huang, J., \& Soderberg, A. M. 2009, ATel, 2263

van Paradijs, J. 1996, ApJ, 464, L139

Webb, N. A., et al. 2000, MNRAS, 317, 528

Wex, N., \& Kopeikin, S. M. 1999, ApJ, 514, 388

Woosley, S. E., Heger, A., \& Weaver, T. A. 2002, Rev. Mod. Phys., 74, 1015

Zhang, W., Woosley, S. E., \& Heger, A. 2008, ApJ, 679, 639

Zurita, C., Casares, J., \& Shahbaz, T. 2003, ApJ, 582, 369

Zurita, C., et al. 2002, MNRAS, 334, 999 